\title{
Analysis of GPS Reflected Signals Based on SNR Measurements: Land Versus Water
}

\author{
Rameez UR Rahman Lighari, Erkki T. Salonen, Markus Berg, Aarno Pärssinen \\ Centre for Wireless Communications - Radio Technology Research Unit, University of Oulu, Finland \\ rameez.lighari@oulu.fi
}

\begin{abstract}
The transmitted Global Positioning System (GPS) signal has Right Hand Circular Polarization (RHCP) and it changes to Left Hand Circular Polarization (LHCP) after being reflected. The proportions of RHCP and LHCP power levels depend on characteristics of reflecting surface and satellite elevation angle. The change of polarization can be evaluated by comparing the measured RHCP and LHCP levels. This paper reports the results of Signal-to-Noise Ratio (SNR) data for direct and reflected GPS signal components measured over sea and land surfaces. First, field measurements with two dual polarized antennas having both RHCP and LHCP are performed in both environments. Then, SNR-based analysis is done to compare reflection levels between two reflecting surfaces. The results show that the SNR of reflected signal from seawater is on average 2 $\mathrm{dB}$ or more higher than that of signal reflected from asphalt or ground.
\end{abstract}

Index Terms - GPS, propagation, measurement, direct signal, reflected signal, circular polarization.

\section{INTRODUCTION}

The reflected signal of Global Positioning System (GPS) contains information about the physical characteristic of reflecting surface. Since the original suggestion by MaritnNeira [1], GPS Reflectometry (GPS-R) technique has been widely used for remote sensing purposes [2]-[5]. Though, the reflected GPS signal may be useful for remote sensing, it also contributes to decreased position accuracy [6]. Furthermore, GPS multipath interference is often associated with areas such as urban canyon and city landscape; however, it is also present in open areas and sea where ground and water serve as reflecting surfaces for GPS signal.

GPS signal has Right Hand Circular Polarization (RHCP) and the incident RHCP signal for the angles less than the Brewster angle produces mainly a Right Hand Circular or Elliptical Polarized (RHCP or RHEP) reflected signal (see Fig. 1). However, at Brewster angle, the resultant reflection is linearly polarized [7]. Generally, incident RHCP signal changes to Left Hand Circular or Elliptical Polarization (LHCP or LHEP) in the reflection for the angles greater than Brewster angle [8]-[9] (see Fig. 1). It should be noted that the reflected signal is always combination of both RHCP and LHCP signals, where one polarized signal is always dominant than other depending on the angle of incidence and Brewster angle. Therefore, the polarization of GPS signal must be taken into account when direct and reflected signal components are

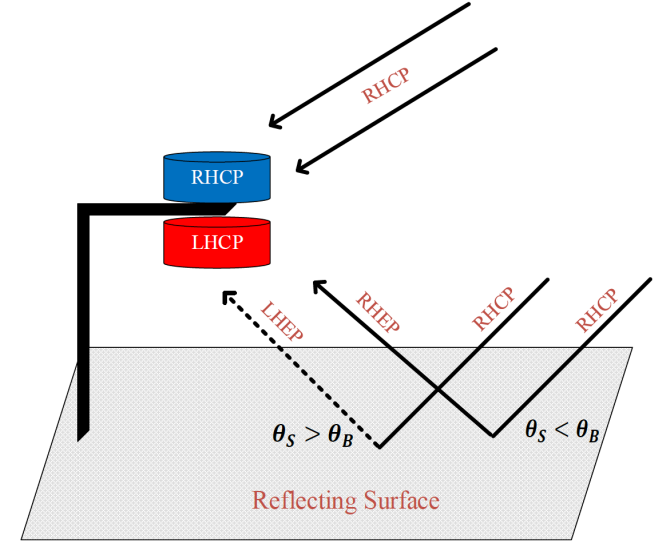

Fig. 1: Typical GPS multipath scenario, where $\theta_{S}$ and $\theta_{B}$ are satellite elevation and Brewster angles, respectively.

recorded simultaneously. The traditional method of recording both direct and reflected GPS signal components consists of two antennas as illustrated in Fig. 1. Direct GPS signals are received by zenith pointing antenna, and is thus RHCP, whereas the nadir pointing antenna is sensitive to LHCP to receive reflected signals, which become LHCP after reflection. The grazing angle of reflected signal corresponds to the incident angle (i.e. satellite elevation angle) of the direct signal. Earlier, we have presented some theoretical calculations to investigate polarization properties of reflected signals in [6], and the fundamental issues of environmental factors, reflection, diffraction and rough surface effects on the GPS signals have been examined in detail by Hannah [7].

The goal of present contribution is to provide an understanding of how the power levels of reflected GPS signal components change from one medium to another; note that the proportions of RHCP and LHCP power levels depend on the conductivity and the dielectric constant of reflecting surface, and the elevation angle of satellite. Final target of the polarization-based investigation is a 3D GPS channel model development for GPS device performance testing in laboratory conditions.

In this paper, Section II briefly discusses properties of reflection coefficients for the circularly polarized wave. In Section III, test setup along with test environments are described. Then, experimental results are presented in Section IV. These results include both the direct and the reflected signal compo- 
nents combined with the instantaneous satellite constellation and the Signal-to-Noise Ratio (SNR) for individual satellites. Finally, Section V concludes the paper.

\section{TheOretical CALCUlations}

For understanding the polarization properties of reflected signals, theoretical calculations for few cases are presented. When a plane wave propagates from air (relative permittivity very close to one) to surface (permittivity, $\varepsilon_{r}$ ) in the incident angle $\theta$, then the reflection coefficients can be calculated separately for horizontal and vertical polarization [7]:

$$
\begin{aligned}
\Gamma_{H} & =\frac{\sin \theta-\sqrt{\varepsilon-\cos ^{2} \theta}}{\sin \theta+\sqrt{\varepsilon-\cos ^{2} \theta}} \\
\Gamma_{V} & =-\frac{\varepsilon \sin \theta-\sqrt{\varepsilon-\cos ^{2} \theta}}{\varepsilon \sin \theta+\sqrt{\varepsilon-\cos ^{2} \theta}} \\
\varepsilon & =\varepsilon_{r}-j 60 \lambda \sigma
\end{aligned}
$$

where $\sigma$ is the conductivity of reflecting surface, $\theta=\theta_{S}$ is satellite elevation angle, and $\lambda$ is the wavelength of $\mathrm{L} 1$ band GPS signal. Then, the resultant reflection can be considered as the sum of two circularly polarized (CP) signal components; the co-polarization (original component) and a cross-polarization (opposite component) [10]. Then, the copolar $\left(\Gamma_{o}\right)$ and the cross-polar $\left(\Gamma_{x}\right)$ reflection coefficients are represented as:

$$
\begin{gathered}
\Gamma_{o}=\frac{\Gamma_{H}+\Gamma_{V}}{2} \\
\Gamma_{x}=\frac{\Gamma_{H}-\Gamma_{V}}{2}
\end{gathered}
$$

Now, the calculation of each circular reflection coefficient is straightforward for any man-made and natural materials. The conductivity and relative permittivity values for materials at GPS L1 frequency, $1.575 \mathrm{GHz}$ are given in Table I [11]-[12]. The electrical properties for seawater are calculated at water temperature of $10^{\circ} \mathrm{C}$ and salinity of $3.5 \mathrm{~g} / \mathrm{kg}$ [12]. Although, in oceans the salinity is 10 times higher, but the measurements were taken in Bay of Bothnia [13], where the salinity level and water temperature is lower than normal oceans. Similarly, for ground (soil) the values were extracted from regression models presented in [11], based on temperature, soil composition and volumetric water content.

The ratio of reflected RHCP signal to transmitted RHCP signal are presented in Fig. 2. The ratio of $0.5(-3 \mathrm{~dB})$ means linear polarization $\left(\theta_{S}\right.$ is Brewster angle) and looking at Fig. 2 it can be seen that Brewster angle varies based on electrical properties of the materials. Furthermore, the magnitude of the cross-polar component increases rapidly beyond Brewster angle and this increase should be directly visible in the SNR of the reflected signal, when compared with the SNR of the direct signal, which is discussed later in Section IV.
TABLE I: ELECTRICAL PROPERTIES OF MATERIALS [11]-[12]

\begin{tabular}{lcc}
\hline \hline Material & Conductivity (S/m) & Relative Permittivity \\
\hline Concrete & $2 \times 10^{-5}$ & 4 \\
Dry Ground & $1 \times 10^{-5}$ & 5 \\
Medium Dry Ground & $4 \times 10^{-2}$ & 8 \\
Seawater & $4 \times 10^{-1}$ & 81 \\
\hline \hline
\end{tabular}

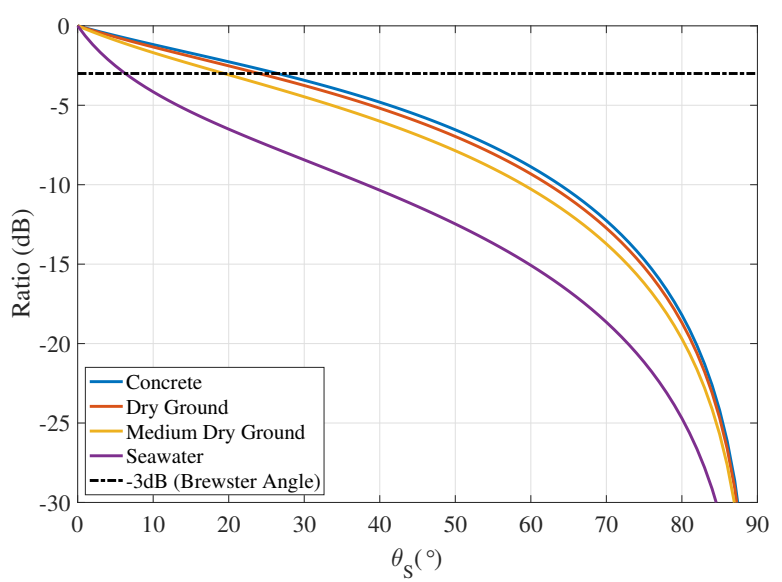

Fig. 2: The ratio of reflected RHCP signal to transmitted RHCP signal at the GPS L1 frequency region. Generally, for the angles less than the Brewster angles the reflected signal has dominant RHEP or more commonly RHCP.

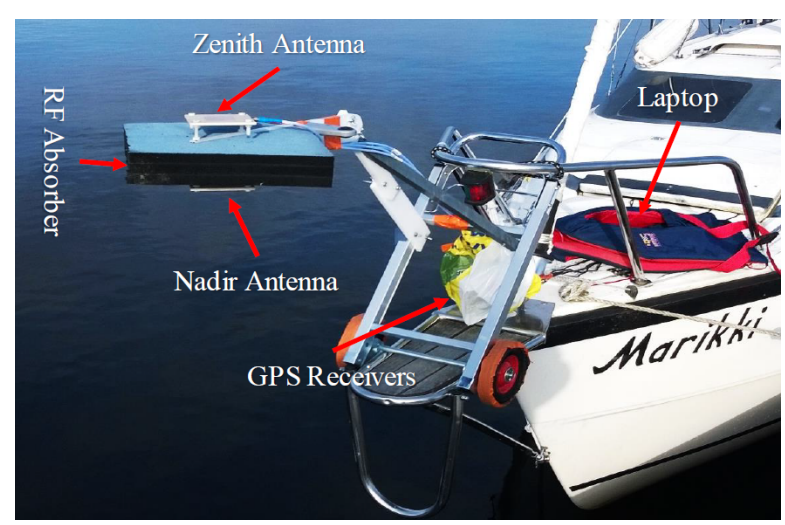

Fig. 3: Photograph of the equipments installed to a sailboat for offshore field tests. Two dual polarized (i.e. RHCP and LHCP) GPS antennas pointing towards zenith and nadir were used to receive direct and reflected signals simultaneously.

\section{FIELD MEASUREMENTS}

\section{A. Data Collection Setup}

Equipment used during experiments consists of two dual polarized GPS antennas for simultaneous LHCP and RHCP reception [14], radio-frequency (RF) absorber, four identical GPS receivers [15], and a laptop for data storing (see Fig. 3). During the tests, receivers collected GPS satellite data at a rate of 1 sample/sec. The GPS antenna has an axial ratio $<2.6$ $\mathrm{dB}$ for both polarizations over the $24 \mathrm{MHz}$ bandwidth at the frequency of $1.575 \mathrm{GHz}$. Details about the GPS antenna used during experiments are described in [14]. 


\section{B. Environments}

Fields test were done to collect dynamic GPS data in two different environments for the analysis. The first field test was carried out in the countryside area with an open swath of land, and 10 minutes worth of data was recorded. The second experiment was done in open sea and yielded 13 minutes worth data.

\section{RESULTS}

For an interference free environment the SNR should be over $40 \mathrm{~dB}$ for Line-of-Sight (LOS) conditions [16]-[19]. However, the SNR of reflected signal is significantly weaker and varies as a function of satellite elevation angle and antenna height above reflecting surface [20].

The distributions of SNR for all tracked satellites are presented in Fig. 4. The difference between the zenith and nadir pointing antennas is evident in countryside test; zenith pointing antenna has slightly skewed SNR distribution for both RHCP and LHCP data as opposed to nadir pointing antenna whose distributions are quite symmetric (see Fig. 4a). Similarly, the histograms of SNR for offshore test show similar trend for zenith pointing antenna with slightly skewed SNR distributions for both RHCP and LHCP data. Even though, RH-SNR data from nadir pointing antenna is quite symmetric, the LH-SNR data is significantly skewed with longer tail (see Fig. 4b) opposed to countryside test; which represents the difference between two reflecting surfaces (e.g. seawater and asphalt/soil). RH-SNR of zenith pointing antenna has similar mean and variance in both environments due to continuous LOS conditions and similar behavior is seen for LH-SNR (see Table II). RH-SNR data collected with nadir pointing antenna has similar variance but mean is approximately $10 \mathrm{~dB}$ lower than that of zenith pointing antenna for countryside test and 7 $\mathrm{dB}$ lower mean for offshore test (see Table II). Similarly, LHSNR of nadir pointing has lower mean and higher variance for both environments compared to zenith pointing antenna and the mean SNR drop is about $2 \mathrm{~dB}$ between two reflecting surfaces (see Table II).

For deeper investigation, the measured cumulative distribution functions (CDFs) of SNR for two satellites from countryside and offshore tests are presented in Fig. 5. Note that the azimuth angle is not considered due to clear sky conditions in both environments. Satellites PRN26 and PRN32 shown in Fig. 5a are located at very low elevation angle of $03^{\circ}$ in sky. Overall results of zenith pointing antenna for countryside test are approximately 3 to $5 \mathrm{~dB}$ lower than that of offshore test and this might be due to satellite being shadowed by distant trees and terrain located in countryside area.

In nadir pointing antenna case, the median value of LHSNR (see Fig. 5a) from offshore test is $2 \mathrm{~dB}$ higher than that of countryside test which correlates with results presented in Table II and similar type of behavior for the LH-SNR (see Table V) of nadir pointing antenna is found between two measured environments. Additionally, when the elevation angle is less than Brewster angle (see Fig. 2) the original
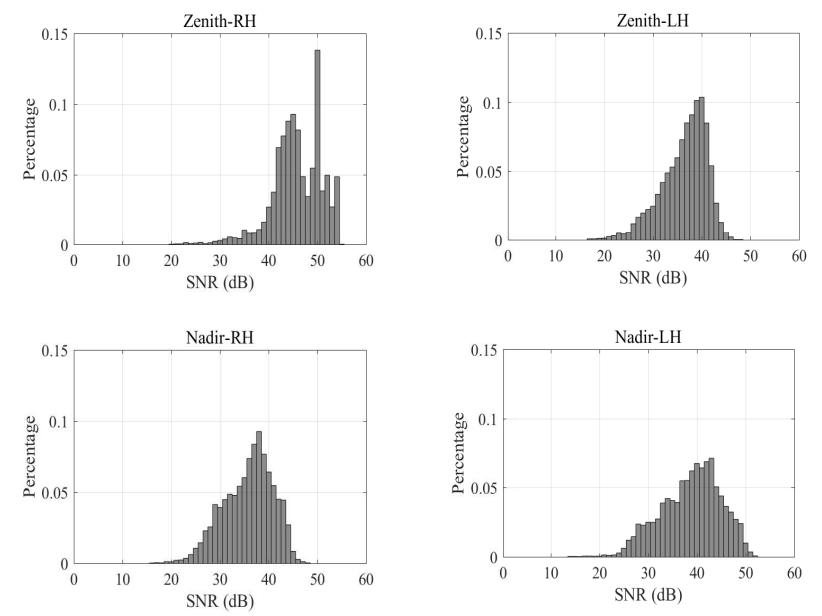

(a) Countryside Test
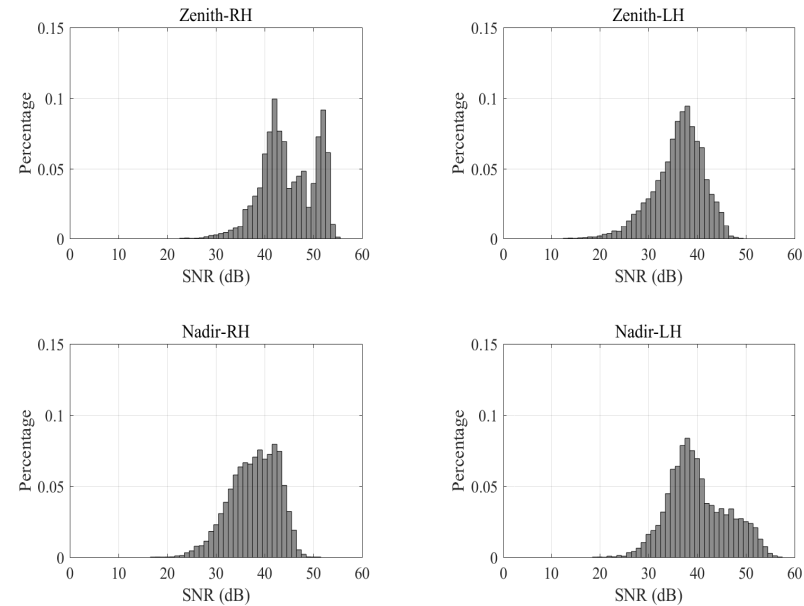

(b) Offshore Test.

Fig. 4: Normalized histograms of SNR measurements. For countryside test (top figure), the reflecting surfaces were consist of asphalt and swath of lands. For offshore test (bottom figure), the reflecting surfaces were consist of mostly seawater and to some extent metal (sailboat).

TABLE II: MEAN AND STANDARD DEVIATION OF SNR

\begin{tabular}{cccccc}
\hline \hline Antenna Port & \multicolumn{2}{c}{ Countryside Test (dB) } & & \multicolumn{2}{c}{ Offshore Test (dB) } \\
\cline { 2 - 3 } \cline { 5 - 6 } & $\boldsymbol{\mu}_{\boldsymbol{S} \boldsymbol{N} \boldsymbol{R}}$ & $\boldsymbol{\sigma}_{\boldsymbol{S} \boldsymbol{N} \boldsymbol{R}}$ & & $\boldsymbol{\mu}_{\boldsymbol{S} \boldsymbol{N} \boldsymbol{R}}$ & $\boldsymbol{\sigma}_{\boldsymbol{S} \boldsymbol{N} \boldsymbol{R}}$ \\
\hline Zenith-RH & 45.81 & 5.15 & & 44.83 & 5.46 \\
Zenith-LH & 36.39 & 4.72 & & 36.22 & 5.07 \\
Nadir-RH & 35.78 & 5.05 & & 37.77 & 4.85 \\
Nadir-LH & 38.78 & 6.11 & & 40.11 & 6.09 \\
\hline \hline
\end{tabular}

RHCP polarization (co-polarized) of the signal is dominant after reflection, and for angles greater than Brewster angle the predominant signal component is the cross-polarized (i.e. LHCP) [21]. This can be visualized in Fig. 5a for nadir pointing antenna, where the RH-SNR is higher than LH-SNR. Similar behavior can be observed from Table $\mathrm{V}$ for lower elevation angles and as the elevation angle increases the LHSNR starts to dominate. 


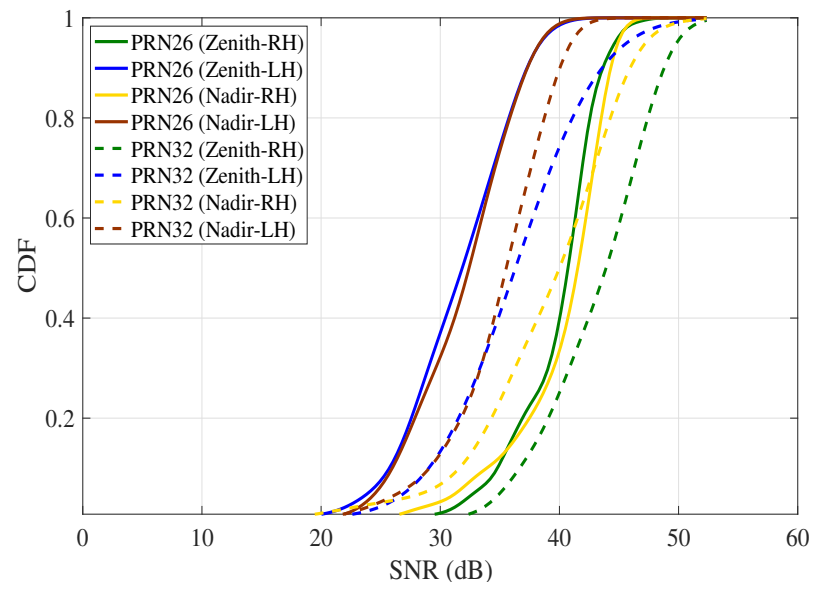

(a) PRN26-Countryside vs PRN32-Offshore.

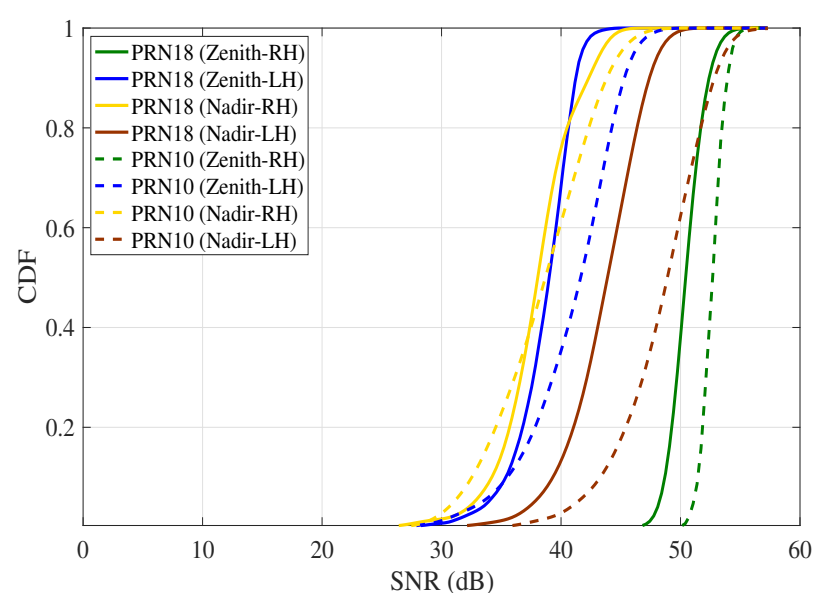

(b) PRN18-Countryside vs PRN10-Offshore.

Fig. 5: Measured CDFs of SNR. The elevation angle of both satellites PRN26 (countryside test) and PRN32 (offshore test) is $03^{\circ}$ (top figure), and the elevation angles of PRN18 (countryside test) and PRN10 (offshore test) satellites are $58^{\circ}$ and $59^{\circ}$, respectively (bottom figure).

Fig. 5b, contains the CDF results of SNR for much higher elevated satellite $\left(>55^{\circ}\right)$. The difference between countryside and offshore is small for zenith pointing antenna. For nadir pointing antenna RH-SNR results are almost identical, whereas, LH-SNR results from offshore test are higher than countryside test, indicating higher reflection coefficient.

For illustration purpose theoretical and measured values for four satellites are compared in Table III. Measured values represent the average difference between the RH-SNR of zenith and nadir pointing antennas, and theoretical values show expected drop of SNR after reflection (see Fig. 2). The values correlate more for offshore test due to precise theoretical calculations. However, for countryside test the difference is due to lack of knowledge about the exact soil composition, which lead to less accurate theoretical results plus other practical impairments. Additionally, the Cross Polarization Discrimination (XPD) level of the receiving antenna has an effect on the measured SNR distributions [14]. For RHCP antenna, the
TABLE III: RH-SNR RATIO BETWEEN ZENITH AND NADIR ANTENNAS

\begin{tabular}{|c|c|c|c|c|c|}
\hline \multirow{2}{*}{$\Uparrow \theta_{S}$} & \multicolumn{2}{|c|}{ Countryside Test $(\mathrm{dB})$} & \multirow{2}{*}{$\Uparrow \boldsymbol{\theta}_{S}$} & \multicolumn{2}{|c|}{ Offshore Test (dB) } \\
\hline & Measured & Theoretical & & Measured & Theoretical \\
\hline 29 & 08 & 04 & 17 & 04 & 06 \\
\hline 41 & 12 & 06 & 27 & 07 & 08 \\
\hline 58 & 12 & 10 & 50 & 15 & 13 \\
\hline 67 & 21 & 11 & 59 & 14 & 15 \\
\hline
\end{tabular}

measured mean XPD value, which is now the LHCP, varies in the range of $11-25 \mathrm{~dB}$ for elevation angles above $0^{\circ}$. For elevation angle $<25^{\circ}$, the XPD for certain azimuth angles drops below $6 \mathrm{~dB}$. Thus, the difference between measured and theoretical SNR behavior for lower elevation angles is a result of the decreased XPD performance of the antenna, and the simplified theoretical model of the nearby measurement environment. Also, the theoretical model does not include the terrain profile for countryside measurements, where the reflections are combination of asphalt and soil, which also lead to mismatch between theory and measurements. Similarly, the resultant reflections during offshore test may not be entirely from seawater due to some contributions from sailboat acting as reflecting surface. Furthermore, detailed SNR statistics (i.e. cumulative percentages of $\mathrm{CDF}$ ) of each tracked satellite for both zenith and nadir pointing antennas are presented in Tables IV, and V, respectively.

\section{CONCLUSIONS}

Two dual polarized (i.e. RHCP and LHCP) GPS antennas pointing towards zenith and nadir were used to record direct and reflected signals simultaneously. The SNR analysis of the dynamic GPS data collected at two different environments has demonstrated that energy levels of reflected GPS signal depend on the satellite elevation angle, and on the characteristics of reflecting surface. Results have demonstrated that the SNR of reflected (LHCP) signal from seawater is on average at least $2 \mathrm{~dB}$ or more higher than that of signal reflected from asphalt and ground. However, more deeper investigation of data is required to interpret more precisely the variables that can influence multipath reflections and SNR outputs.

\section{REFERENCES}

[1] M. Maritn-Neira, "A Passive Reflectometry and Interferometry System (PARIS): Application to Ocean Altimetry," ESA Journal, vol. 17, no. 4, pp. 331-355, 1993.

[2] A. Rius, E. Cardellach, and M. Martin-Neira, "Altimetric Analysis of the Sea-surface GPS-Reflected Signals," IEEE Trans. Geosci. Remote Sens., vol. 48, no. 4, pp. 2119-2127, Apr. 2010.

[3] N. Rodriguez-Alvarez, X. Bosch-Lluis, A. Camps, M. Vall-llossera, E. Valencia, J. F. Marchan-Hernandez, and I. Ramos-Perez, "Soil Moisture Retrieval Using GNSS-R Techniques: Experimental Results Over a Bare Soil Field," IEEE Trans. Geosci. Remote Sens., vol. 47, no. 11, pp. 36163624, Nov. 2009.

[4] A. Komjathy, J. Maslanik, V. U. Zavorotny, P. Axelrad, and S. J Katzberg, "Sea Ice Remote Sensing Using Surface Reflected GPS Signals," Proc. IEEE IGARSS, pp. 2855-2857, Honolulu, HI, Jul. 2000.

[5] V. U. Zavorotny and A. G. Voronovich, "Scattering of GPS Signals from the Ocean with Wind Remote Sensing Application," IEEE Trans. Geosci. Remote Sens., vol. 38, no. 2, pp. 951-964, Mar. 2000. 
TABLE IV: STATISTICS OF SNR FOR ZENITH POINTING ANTENNA

\begin{tabular}{|c|c|c|c|c|c|c|c|c|c|c|c|c|c|c|c|}
\hline \multicolumn{9}{|c|}{ Countryside Test } & \multicolumn{7}{|c|}{ Offshore Test } \\
\hline \multirow{2}{*}{$\Uparrow \boldsymbol{\theta}_{S}$} & \multirow{2}{*}{ Satellite } & \multicolumn{3}{|c|}{ RH-SNR (dB) } & \multicolumn{3}{|c|}{ LH-SNR (dB) } & \multirow{2}{*}{$\Uparrow \theta_{S}$} & \multirow{2}{*}{ Satellite } & \multicolumn{3}{|c|}{ RH-SNR (dB) } & \multicolumn{3}{|c|}{ LH-SNR (dB) } \\
\hline & & $10 \%$ & $50 \%$ & $90 \%$ & $10 \%$ & $50 \%$ & $90 \%$ & & & $10 \%$ & $50 \%$ & $90 \%$ & $10 \%$ & $50 \%$ & $90 \%$ \\
\hline 01 & PRN11 & 29 & 37 & 45 & 23 & 37 & 41 & 03 & PRN32 & 37 & 44 & 48 & 29 & 37 & 43 \\
\hline 03 & PRN26 & 35 & 41 & 44 & 26 & 32 & 37 & 04 & PRN07 & 32 & 41 & 44 & 27 & 36 & 39 \\
\hline 11 & PRN07 & 35 & 41 & 43 & 27 & 35 & 39 & 07 & PRN28 & 34 & 38 & 41 & 26 & 35 & 39 \\
\hline 17 & PRN30 & 41 & 43 & 45 & 31 & 38 & 40 & 10 & PRN11 & 38 & 42 & 44 & 28 & 36 & 41 \\
\hline 20 & PRN13 & 42 & 45 & 47 & 31 & 39 & 42 & 13 & PRN30 & 40 & 41 & 43 & 30 & 35 & 38 \\
\hline 21 & PRN20 & 42 & 44 & 45 & 31 & 37 & 40 & 14 & PRN20 & 40 & 43 & 45 & 33 & 38 & 41 \\
\hline 25 & PRN16 & 45 & 46 & 47 & 31 & 38 & 42 & 15 & PRN13 & 41 & 43 & 45 & 29 & 37 & 41 \\
\hline 29 & PRN15 & 43 & 45 & 47 & 30 & 39 & 42 & 17 & PRN16 & 40 & 42 & 44 & 29 & 36 & 41 \\
\hline 37 & PRN21 & 47 & 49 & 50 & 31 & 37 & 41 & 25 & PRN21 & 46 & 47 & 48 & 33 & 39 & 42 \\
\hline 41 & PRN08 & 48 & 50 & 51 & 28 & 34 & 38 & 27 & PRN15 & 46 & 48 & 49 & 32 & 42 & 45 \\
\hline 46 & PRN10 & 50 & 51 & 52 & 37 & 40 & 44 & 50 & PRN08 & 50 & 50 & 51 & 27 & 34 & 39 \\
\hline 58 & PRN18 & 50 & 50 & 52 & 35 & 39 & 41 & 56 & PRN18 & 51 & 51 & 52 & 31 & 37 & 42 \\
\hline 67 & PRN27 & 53 & 54 & 54 & 34 & 40 & 43 & 59 & PRN10 & 52 & 53 & 54 & 36 & 42 & 45 \\
\hline- & - & - & - & - & - & - & - & 64 & PRN27 & 52 & 52 & 53 & 33 & 39 & 43 \\
\hline
\end{tabular}

TABLE V: STATISTICS OF SNR FOR NADIR POINTING ANTENNA

\begin{tabular}{|c|c|c|c|c|c|c|c|c|c|c|c|c|c|c|c|}
\hline \multicolumn{9}{|c|}{ Countryside Test } & \multicolumn{7}{|c|}{ Offshore Test } \\
\hline \multirow{2}{*}{$\Uparrow \theta_{S}$} & \multirow{2}{*}{ Satellite } & \multicolumn{3}{|c|}{ RH-SNR (dB) } & \multicolumn{3}{|c|}{ LH-SNR (dB) } & \multirow{2}{*}{$\Uparrow \boldsymbol{\theta}_{S}$} & \multirow{2}{*}{ Satellite } & \multicolumn{3}{|c|}{ RH-SNR (dB) } & \multicolumn{3}{|c|}{ LH-SNR (dB) } \\
\hline & & $10 \%$ & $50 \%$ & $90 \%$ & $10 \%$ & $50 \%$ & $90 \%$ & & & $10 \%$ & $50 \%$ & $90 \%$ & $10 \%$ & $50 \%$ & $90 \%$ \\
\hline 01 & PRN11 & 24 & 39 & 44 & 26 & 38 & 42 & 03 & PRN32 & 32 & 40 & 45 & 29 & 35 & 40 \\
\hline 03 & PRN26 & 34 & 42 & 44 & 26 & 33 & 37 & 04 & PRN07 & 34 & 44 & 46 & 30 & 34 & 38 \\
\hline 11 & PRN07 & 35 & 40 & 43 & 27 & 33 & 38 & 07 & PRN28 & 34 & 39 & 43 & 31 & 35 & 38 \\
\hline 17 & PRN30 & 26 & 33 & 40 & 28 & 37 & 42 & 10 & PRN11 & 35 & 41 & 44 & 34 & 38 & 41 \\
\hline 20 & PRN13 & 29 & 35 & 39 & 34 & 39 & 42 & 13 & PRN30 & 34 & 42 & 44 & 35 & 39 & 42 \\
\hline 21 & PRN20 & 27 & 33 & 38 & 28 & 36 & 40 & 14 & PRN20 & 29 & 37 & 43 & 29 & 36 & 40 \\
\hline 25 & PRN16 & 29 & 35 & 41 & 31 & 38 & 44 & 15 & PRN13 & 30 & 36 & 42 & 35 & 39 & 42 \\
\hline 29 & PRN15 & 31 & 37 & 40 & 38 & 43 & 45 & 17 & PRN16 & 31 & 38 & 41 & 34 & 39 & 42 \\
\hline 37 & PRN21 & 33 & 38 & 43 & 39 & 42 & 44 & 25 & PRN21 & 28 & 33 & 38 & 33 & 37 & 42 \\
\hline 41 & PRN08 & 29 & 38 & 42 & 36 & 41 & 46 & 27 & PRN15 & 33 & 41 & 44 & 37 & 44 & 47 \\
\hline 46 & PRN10 & 28 & 32 & 41 & 30 & 44 & 49 & 50 & PRN08 & 30 & 35 & 40 & 42 & 47 & 51 \\
\hline 58 & PRN18 & 34 & 38 & 42 & 39 & 44 & 47 & 56 & PRN18 & 30 & 35 & 41 & 38 & 44 & 48 \\
\hline 67 & PRN27 & 27 & 33 & 37 & 42 & 47 & 50 & 59 & PRN10 & 33 & 39 & 44 & 43 & 49 & 53 \\
\hline- & - & - & - & - & - & - & - & 64 & PRN27 & 32 & 37 & 42 & 44 & 50 & 53 \\
\hline
\end{tabular}

[6] R. Lighari, M. Berg, J. Kallankari, A. Parssinen, and E. T. Salonen, "Analysis of the Measured RHCP and LHCP GNSS Signals in Multipath Environment," in 6th International Conference on Localization and GNSS (ICL-GNSS), Barcelona, Spain, Jun. 2016.

[7] B. M. Hannah, "Modelling and Simulation of GPS Multipath Propagation," Doctoral dissertation, Queensland University of Technology, 2001.

[8] P. D. Groves, "GNSS Solutions: Multipath vs. NLOS Signals. How Does Non-Line-of-Sight Reception Differ from Multipath Interference," Inside GNSS Magazine, Vol. 8, no. 6, pp. 40-42, 2013.

[9] U. Inan, and A. Inan, "Electromagnetic Waves," Prentice Hall, 2000.

[10] S. Saunders, and A. Aragn-Zavala, "Antennas and Propagation for Wireless Communication Systems," 2nd Edition, John Wiley \& Sons, 2007.

[11] ITU-R, "Electrical Characteristics of the Surface of the Earth," Rec P.527-4, Jun. 2017.

[12] L. A. Klein, and C. T. Swift "An Improved Model for the Dielectric Constant of Sea Water at Microwave Frequencies," IEEE J. Ocean. Eng., Vol. 2, no. 1, pp. 104-111, Jan. 1977.

[13] Bothnian Bay, [online], Available: http://www.visitbothnianbay.com/ (Accessed: Oct. 10, 2017).

[14] M. Berg, R. Lighari, T. Tuovinen, and E. T. Salonen, "Circularly Polarized GPS Antenna for Simultaneous LHCP and RHCP Reception with
High Isolation," in Loughborough Antennas and Propagation Conference (LAPC), Loughborough, UK, Nov. 2016.

[15] U-blox Holding AG, "EVK-7 U-blox 7 GNSS Evaluation Kits," [online], Available: https://www.u-blox.com/en/product/evk-7 (Accessed: Oct. 10, 2017).

[16] E. D. Kaplan, and C. J. Hegarty, 'Understanding GPS: Principles and Applications," 2nd Edition, Artech House, 2006.

[17] P. D. Groves, "Principles of GNSS, Inertial, and Multisensor Integrated Navigation Systems," 2nd Edition, Artech House, 2013.

[18] Z. He, M. Petovello, and G. Lachapelle, "Indoor Doppler Error Characterization for High Sensitivity GNSS Receivers," IEEE Trans. Aerosp. Electron. Syst., vol. 50, no. 3, pp. 2185-2198, 2014.

[19] A. Bilich, P. Axelrad, and K. M. Larson, "Scientific Utility of the Signal-to-Noise Ratio (SNR) Reported by Geodetic GPS Receivers," Proceedings of ION GNSS, pp. 1999-2010, Sept. 2007.

[20] K. M. Larson, J. S. Löfgren, and R. Haas, "Coastal Sea Level Measurements Using a Single Geodetic GPS Receiver," Adv. Sp. Res., vol. 51, no. 8, pp. 1301-1310, Apr. 2013.

[21] J. S. Löfgren, H. Rüdiger , HG. Scherneck, and M. S. Bos, "Three Months of Local Sea Level Derived from Reflected GNSS Signals," Radio Science, vol. 46, no. 6, 2011. 